Kragujevac Journal of Mathematics

Volume 42(4) (2018), Pages 631-641.

\title{
IDEALS OF IS-ALGEBRAS BASED ON $\mathcal{N}$-STRUCTURES
}

\author{
HASHEM BORDBAR ${ }^{1 *}$, MOHAMMAD MEHDI ZAHEDI ${ }^{2}$, AND YOUNG BAE JUN ${ }^{3}$
}

\begin{abstract}
The notion of a left (resp., right) $\mathcal{N}_{\mathcal{J}}$-ideal is introduced, and related properties are investigated. Characterizations of a left (resp., right) $\mathcal{N}_{\mathcal{J}}$-ideal are considered. Translations of a left (resp., right) $\mathcal{N}_{\mathcal{J}}$-ideal are studied. We show that the homomorphic image (preimage) of a left (resp., right) $\mathcal{N}_{\mathcal{J}}$-ideal is a left (resp., right) $\mathcal{N}_{\mathcal{J}}$-ideal. The notion of retrenched left (resp., right) $\mathcal{N}_{\mathcal{J}}$-ideals is introduced, and their properties are investigated.
\end{abstract}

\section{INTRODUCTION}

Most of the generalization of the crisp set have been conducted on the unit interval $[0,1]$ and they are consistent with the asymmetry observation because a (crisp) set $A$ in a universe $X$ can be defined in the form of its characteristic function $\mu_{A}: X \rightarrow\{0,1\}$ yielding the value 1 for elements belonging to the set $A$ and the value 0 for elements excluded from the set $A$. In other words, the generalization of the crisp set to fuzzy sets relied on spreading positive information that fit the crisp point $\{1\}$ into the interval $[0,1]$. Because no negative meaning of information is suggested, we now feel a need to deal with negative information. To do so, we also feel a need to supply mathematical tool. To attain such object, Jun et al. [3] introduced a new function which is called negative-valued function, and constructed $\mathcal{N}$-structures. They applied $\mathcal{N}$-structures to BCK/BCI-algebras, and discussed $\mathcal{N}$-subalgebras and $\mathcal{N}$-ideals in $\mathrm{BCK} / \mathrm{BCI}$-algebras. The $\mathcal{N}$-structures are applied to $B E$-algebras and subtraction algebras (see [1] and [5]).

In this paper, using the $\mathcal{N}$-structures, we introduce the notion of a left (resp., right)

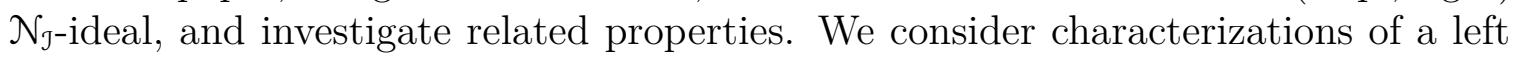

Key words and phrases. Left (resp., right) $\mathcal{N}_{\mathcal{J}}$-ideal, translation, closed support, retrenched left (resp., right) $\mathcal{N}_{\mathcal{J}}$-ideal.

2010 Mathematics Subject Classification. Primary: 06F35. Secondary: 03G25, 03B52.

Received: November 06, 2016.

Accepted: August 26, 2017. 


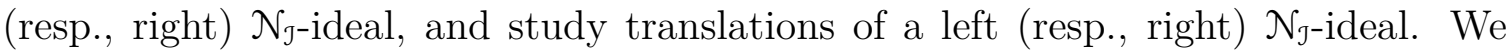
show that the homomorphic image (preimage) of a left (resp., right) $\mathcal{N}_{\mathcal{J}}$-ideal is a left (resp., right) $\mathcal{N}_{\mathcal{J}}$-ideal. We also introduction the notion of retrenched left (resp., right)

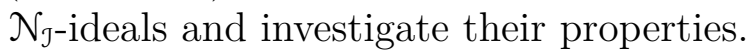

\section{Preliminaries}

Let $K(\tau)$ be the class of all algebras with type $\tau=(2,0)$. By a BCI-algebra we mean a system $X:=(X, *, \theta) \in K(\tau)$ in which the following axioms hold:

(i) $((x * y) *(x * z)) *(z * y)=\theta$;

(ii) $(x *(x * y)) * y=\theta$;

(iii) $x * x=\theta$;

(iv) $x * y=y * x=\theta \Rightarrow x=y$;

for all $x, y, z \in X$. If a BCI-algebra $X$ satisfies $\theta * x=\theta$ for all $x \in X$, then we say that $X$ is a $B C K$-algebra. We can define a partial ordering $\preceq$ by

$$
(\forall x, y \in X)(x \preceq y \Rightarrow x * y=\theta) .
$$

In a BCK/BCI-algebra $X$, the following hold:

$$
\begin{aligned}
& (\forall x \in X)(x * \theta=x), \\
& (\forall x, y, z \in X)((x * y) * z=(x * z) * y) .
\end{aligned}
$$

A subset $I$ of a $B C K / B C I$-algebra $X$ is called an ideal of $X$ if it satisfies

(I1) $0 \in I$;

(I2) $(\forall x, y \in X)(x * y \in I, y \in I \Rightarrow x \in I)$.

We refer the reader to the books [2] and [6] for further information regarding BCK/BCI-algebras.

An IS-algebra (see [4]) is a non-empty set $X$ with two binary operations "*" and "." and constant $\theta$ satisfying the conditions:

- $I(X):=(X, *, \theta)$ is a $B C I$-algebra;

- $S(X):=(X, \cdot)$ is a semigroup;

- the operation "." is distributive (on both sides) over the operation "*", that is,

$$
x \cdot(y * z)=(x \cdot y) *(x \cdot z) \text { and }(x * y) \cdot z=(x \cdot z) *(y \cdot z),
$$

for all $x, y, z \in X$.

In an IS-algebra $X$, the following hold:

$$
\begin{aligned}
& (\forall x \in X)(\theta x=x \theta=\theta) ; \\
& (\forall x, y, z \in X)(x \preceq y \Rightarrow x z \preceq y z, z x \preceq z y) .
\end{aligned}
$$

In what follows we use the notation $x y$ instead of $x \cdot y$.

A nonempty subset $A$ of an IS-algebra $X$ is called a left (resp., right) J-ideal of $X$ (see [4]) if 
(i) $A$ is a left (resp., right) stable subset of $S(X)$, that is, $x a \in A$ (resp., $a x \in A$ ) whenever $x \in S(X)$ and $a \in A$;

(ii) $(\forall x, y \in I(X))(x * y \in A, y \in A \Rightarrow x \in A)$.

For any family $\left\{a_{i} \mid i \in \Lambda\right\}$ of real numbers, we define

$$
\begin{aligned}
& \bigvee\left\{a_{i} \mid i \in \Lambda\right\}:= \begin{cases}\max \left\{a_{i} \mid i \in \Lambda\right\}, & \text { if } \Lambda \text { is finite, } \\
\sup \left\{a_{i} \mid i \in \Lambda\right\}, & \text { otherwise. }\end{cases} \\
& \bigwedge\left\{a_{i} \mid i \in \Lambda\right\}:= \begin{cases}\min \left\{a_{i} \mid i \in \Lambda\right\}, & \text { if } \Lambda \text { is finite, } \\
\inf \left\{a_{i} \mid i \in \Lambda\right\}, & \text { otherwise. }\end{cases}
\end{aligned}
$$

\section{Ideals Based on $\mathcal{N}$-STRuctures}

Denote by $\mathcal{F}(X,[-1,0])$ the collection of functions from a set $X$ to $[-1,0]$. We say that an element of $\mathcal{F}(X,[-1,0])$ is a negative-valued function from $X$ to $[-1,0]$ (briefly, $\mathcal{N}$-function on $X$ ). By an $\mathcal{N}$-structure we mean an ordered pair $(X, f)$ of $X$ and an $\mathcal{N}$-function $f$ on $X$. In what follows, let $X$ denote an IS-algebra unless otherwise specified.

Definition 3.1. An $\mathcal{N}$-structure $(X, f)$ is said to satisfy the left (resp., right) condition in $S(X)$ if $f(x y) \leq f(y)$ (resp., $f(x y) \leq f(x)$ ) for all $x$ and $y$ in $S(X)$.

Definition 3.2. An $\mathcal{N}$-structure $(X, f)$ is called a left (resp., right) $\mathcal{N}_{\mathcal{J}}$-ideal of $X$ if $(X, f)$ satisfies the left (resp., right) condition in $S(X)$ and

$$
(\forall x, y \in X)(f(\theta) \leq f(x) \leq \bigvee\{f(x * y), f(y)\})
$$

Example 3.1. Define two binary operations "*" and "." on a set $X=\{\theta, a, b, c\}$ as follows:

\begin{tabular}{l|lllll|llll}
$*$ & $\theta$ & $a$ & $b$ & $c$ & $\cdot$ & $\theta$ & $a$ & $b$ & $c$ \\
\hline$\theta$ & $\theta$ & $\theta$ & $c$ & $b$ & $\theta$ & $\theta$ & $\theta$ & $\theta$ & $\theta$ \\
$a$ & $a$ & $\theta$ & $c$ & $b$ & $a$ & $\theta$ & $\theta$ & $\theta$ & $\theta$ \\
$b$ & $b$ & $b$ & $\theta$ & $c$ & $b$ & $\theta$ & $\theta$ & $b$ & $c$ \\
$c$ & $c$ & $c$ & $b$ & $\theta$ & $c$ & $\theta$ & $\theta$ & $c$ & $b$
\end{tabular}.

Then $X$ is an IS-algebra (see $[4])$. Let $(X, f)$ be an $\mathcal{N}$-structure in which $f$ is given as follows:

$$
f=\left(\begin{array}{cccc}
\theta & a & b & c \\
-0.8 & -0.6 & -0.3 & -0.3
\end{array}\right) .
$$

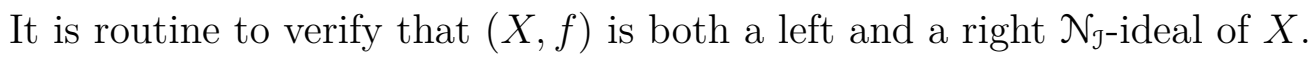

We provide characterizations of a left (resp., right) $\mathcal{N}_{\mathcal{J}}$-ideal.

Theorem 3.1. An $\mathcal{N}$-structure $(X, f)$ is a left $\mathcal{N}_{\mathcal{J}}$-ideal of $X$ if and only if the following assertions are valid

$$
\begin{aligned}
& (\forall x, y \in X) \quad(f(x y) \leq f(y)) \\
& (\forall x, y \in X) \quad(f(x) \leq \bigvee\{f(x * y), f(y)\})
\end{aligned}
$$


Proof. The necessity is clear. Assume that $(X, f)$ satisfies two conditions (3.2) and (3.3). Using (2.3) and (3.2) induce $f(\theta)=f(\theta y) \leq f(y)$ for all $y \in X$. Hence $(X, f)$ is a left $\mathcal{N}_{\mathcal{J}}$-ideal of $X$.

Similarly we have the following theorem.

Theorem 3.2. An $\mathcal{N}$-structure $(X, f)$ is a right $\mathcal{N}_{\mathcal{J}}$-ideal of $X$ if and only if $f$ satisfies the condition (3.3) and

$$
(\forall x, y \in X) \quad(f(x y) \leq f(x)) .
$$

For any $\mathcal{N}$-structure $(X, f)$ and $t \in[-1,0)$, the set

$$
C(f ; t):=\{x \in X \mid f(x) \leq t\}
$$

is called a closed t-support of $(X, f)$ (see [3]).

Theorem 3.3. If an $\mathcal{N}$-structure $(X, f)$ is a left $\mathcal{N}_{\mathcal{J}}$-ideal of $X$, then the closed $t$ support of $(X, f)$ is a left J-ideal of $X$ for all $t \in[f(\theta), 0]$.

Proof. Let $x \in S(X)$ and $a \in C(f ; t)$ for $t \in[f(\theta), 0]$. Then $f(a) \leq t$, and so $f(x a) \leq$ $f(a) \leq t$ which shows that $x a \in C(f ; t)$. It follows from $(2.3)$ that $\theta=\theta a \in C(f ; t)$. Let $x, y \in X$ be such that $x * y \in C(f ; t)$ and $y \in C(f ; t)$ for $t \in[f(\theta), 0]$. Then $f(x * y) \leq t$ and $f(y) \leq t$. It follows from (3.3) that

$$
f(x) \leq \bigvee\{f(x * y), f(y)\} \leq t
$$

and so that $x \in C(f ; t)$. Therefore $C(f ; t)$ is an J-ideal of $X$ for all $t \in[f(\theta), 0]$.

Theorem 3.4. If an $\mathcal{N}$-structure $(X, f)$ is a right $\mathcal{N}_{\mathcal{J}}$-ideal of $X$, then the closed $t$-support of $(X, f)$ is a right J-ideal of $X$ for all $t \in[f(\theta), 0]$.

Proof. It is similar to the proof of Theorem 3.3.

Theorem 3.5. Given an $\mathcal{N}$-structure $(X, f)$, if the nonempty closed $t$-support of $(X, f)$ is a left $\mathcal{J}$-ideal of $X$ for all $t \in[-1,0)$, then $(X, f)$ is a left $\mathcal{N}_{\mathcal{J}}$-ideal of $X$.

Proof. Assume that $C(f ; t)$ is a left J-ideal of $X$ for all $t \in[-1,0)$ with $C(f ; t) \neq \emptyset$. If $f(a b)>f(b)$ for some $a, b \in X$, then there exists $t \in[-1,0)$ such that $f(a b)>t \geq f(b)$. It follows that $b \in C(f ; t)$ and $a b \notin C(f ; t)$, which is a contradiction. Hence $(3.2)$ is valid. Now suppose that (3.3) is false. Then there exists $a, b \in X$ such that

$$
f(a)>\bigvee\{f(a * b), f(b)\} .
$$

Taking $t:=\frac{1}{2}(f(a)+\bigvee\{f(a * b), f(b)\})$ implies that $a * b \in C(f ; t), b \in C(f ; t)$ and $a \notin C(f ; t)$. This is a contradiction, and so $(3.3)$ is valid. Therefore $(X, f)$ is a left $\mathcal{N}_{\mathcal{J}}$-ideal of $X$ by Theorem 3.1.

Similarly we have the following theorem.

Theorem 3.6. Given an $\mathcal{N}$-structure $(X, f)$, if the nonempty closed t-support of $(X, f)$ is a right $\mathcal{J}$-ideal of $X$ for all $t \in[-1,0)$, then $(X, f)$ is a right $\mathcal{N}_{\mathcal{J}}$-ideal of $X$. 
Theorem 3.7. For any left J-ideal $A$ of $X$ and any fixed number $t$ in an open interval $(-1,0)$, there exists a left $\mathcal{N}_{\mathcal{J}}$-ideal $(X, f)$ of $X$ on which $A$ is the closed $t$-support of $(X, f)$.

Proof. Let $(X, f)$ be an $\mathcal{N}$-structure on which $f$ is given as follows:

$$
f(x)= \begin{cases}t, & \text { if } x \in A, \\ 0, & \text { if } x \notin A .\end{cases}
$$

Let $x, y \in X$. If $y \notin A$, then $f(y)=0$ and thus

$$
f(x) \leq 0=\bigvee\{f(x * y), f(y)\} .
$$

Assume that $y \in A$. If $x \in A$, then $x * y$ may or may not belong to $A$. In any case, we have

$$
f(x) \leq \bigvee\{f(x * y), f(y)\} .
$$

If $x \notin A$, then $x * y \notin A$ and hence

$$
f(x)=0=\bigvee\{f(x * y), f(y)\} .
$$

For any $x, y \in X$, if $y \in A$ then $x y \in A$. Hence $f(x y)=t=f(y)$. If $y \notin A$, then $f(y)=0$ and so $f(x y) \leq 0=f(y)$. It follows from Theorem 3.1 that $(X, f)$ is a left $\mathcal{N}_{\mathcal{J}}$-ideal of $X$. Obviously, $A=C(f ; t)$.

Similarly, we have the following theorem.

Theorem 3.8. For any right J-ideal $A$ of $X$ and any fixed number $t$ in an open interval $(-1,0)$, there exists a right $\mathcal{N}_{\mathcal{J}}$-ideal $(X, f)$ of $X$ on which $A$ is the closed t-support of $(X, f)$.

Theorem 3.9. For any nonempty subset $A$ of $X$ and $t \in[-1,0)$, let $(X, f)$ be an $\mathcal{N}$-structure on which $f$ is given as follows:

$$
f(x)= \begin{cases}t, & \text { if } x \in A, \\ 0, & \text { if } x \notin A .\end{cases}
$$

If $A$ is a left (resp., right) J-ideal of $X$, then $(X, f)$ is a left (resp., right) $\mathcal{N}_{\mathcal{J}}$-ideal of $X$.

Proof. Suppose that $A$ is a left J-ideal of $X$. Let $x, y \in X$. If $y \in A$, then $x y \in A$, and

(i) $x * y$ may or may not belong to $A$ whenever $x \in A$;

(ii) $x * y \notin A$ whenever $x \notin A$.

Hence $f(x y)=t=f(y)$ and $f(x * y) \leq \bigvee\{f(x * y), f(y)\}$. If $y \notin A$, then $f(x y) \leq$ $0=f(y)$ and $f(x * y) \leq 0=\bigvee\{f(x * y), f(y)\}$. Therefore $(X, f)$ is a left $\mathcal{N}_{\mathcal{J}}$-ideal of $X$ by Theorem 3.1. Similarly we can prove it for the right case.

Corollary 3.1. For any nonempty subset $A$ of $X$ and an $\mathcal{N}$-structure $(X, f)$ with $\operatorname{Im}(f)=\{-1,0\}$, the following assertions are equivalent. 
(1) $A$ is a left (resp., right) J-ideal of $X$.

(2) $(X, f)$ is a left (resp., right) $\mathcal{N}_{\mathcal{J}}$-ideal of $X$.

Theorem 3.10. If an $\mathcal{N}$-structure $(X, f)$ is a left (resp., right) $\mathcal{N}_{\mathcal{J}}$-ideal of $X$, then the set

$$
X_{f}:=\{x \in X \mid f(x)=f(\theta)\}
$$

is a left (resp., right) J-ideal of $X$.

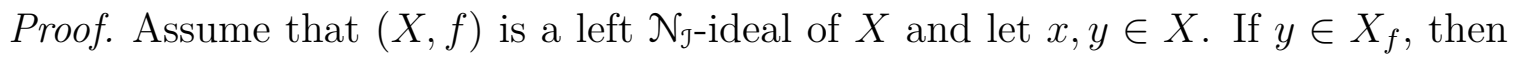
$f(x y) \leq f(y)=f(\theta)$ and so $f(x y)=f(\theta)$, that is, $x y \in X_{f}$. Obviously, $\theta \in X_{f}$. Suppose that $x * y \in X_{f}$ and $y \in X_{f}$. Then

$$
f(x) \leq \bigvee\{f(x * y), f(y)\}=f(\theta),
$$

and so $f(x)=f(\theta)$, i.e., $x \in X_{f}$. Therefore $X_{f}$ is a left J-ideal of $X$. Similarly, we can prove it for the right case.

Given an $\mathcal{N}$-structure $(X, f)$, we denote

$$
\perp:=-1-\bigwedge\{f(x) \mid x \in X\} .
$$

For any $\alpha \in[\perp, 0]$, the $\alpha$-translation of $(X, f)$ is defined to be the new $\mathcal{N}$-structure $\left(X, f_{\alpha}\right)$ on which $f_{\alpha}$ is defined by $f_{\alpha}(x)=f(x)+\alpha$ for all $x \in X$.

Theorem 3.11. For every $\alpha \in[\perp, 0]$, the $\alpha$-translation of a left (resp., right) $\mathcal{N}_{\mathcal{J}}$-ideal is a left (resp., right) $\mathcal{N}_{\mathcal{J}}$-ideal of $X$.

Proof. Let $\alpha \in[\perp, 0]$ and let $(X, f)$ be a left $\mathcal{N}_{\mathcal{J}}$-ideal of $X$. For any $x, y \in X$, we have $f_{\alpha}(x y)=f(x y)+\alpha \leq f(y)+\alpha=f_{\alpha}(y)$ and

$$
\begin{aligned}
f_{\alpha}(x) & =f(x)+\alpha \leq \bigvee\{f(x * y), f(y)\}+\alpha \\
& =\bigvee\{f(x * y)+\alpha, f(y)+\alpha\} \\
& =\bigvee\left\{f_{\alpha}(x * y), f_{\alpha}(y)\right\} .
\end{aligned}
$$

It follows from Theorem 3.1 that $\left(X, f_{\alpha}\right)$ is a left $\mathcal{N}_{\mathcal{J}}$-ideal of $X$. For the right case, it is similar.

Theorem 3.12. For $\mathcal{N}$-structure $(X, f)$, if there exists $\alpha \in[\perp, 0]$ such that every $\alpha$-translation of $(X, f)$ is a left (resp., right) $\mathcal{N}_{\mathcal{J}}$-ideal, then $(X, f)$ is a left (resp., right) $\mathcal{N}_{\mathcal{J}}$-ideal of $X$.

Proof. Assume that the $\alpha$-translation $\left(X, f_{\alpha}\right)$ of $(X, f)$ is a left $\mathcal{N}_{\mathcal{J}}$-ideal of $X$. For any $x, y \in X$, we have $f(x y)+\alpha=f_{\alpha}(x y) \leq f_{\alpha}(y)=f(y)+\alpha$ and

$$
\begin{aligned}
f(x)+\alpha & =f_{\alpha}(x) \leq \bigvee\left\{f_{\alpha}(x * y), f_{\alpha}(y)\right\} \\
& =\bigvee\{f(x * y)+\alpha, f(y)+\alpha\} \\
& =\bigvee\{f(x * y), f(y)\}+\alpha
\end{aligned}
$$


It follows that $f(x y) \leq f(y)$ and $f(x) \leq \bigvee\{f(x * y), f(y)\}$. Therefore $(X, f)$ is a left $\mathcal{N}_{\mathcal{J}}$-ideal of $X$ by Theorem 3.1 .

For any $\mathcal{N}$-structure $(X, f), \alpha \in[\perp, 0]$ and $t \in[-1, \alpha)$, the set

$$
C_{\alpha}(f ; t):=\{x \in X \mid f(x) \leq t-\alpha\}
$$

is called the $\alpha$-translation of closed $t$-support of $(X, f)$

Theorem 3.13. Let $(X, f)$ be an $\mathcal{N}$-structure and $\alpha \in[\perp, 0]$. If $(X, f)$ is a left (resp., right) $\mathcal{N}_{\mathcal{J}}$-ideal of $X$, then the $\alpha$-translation of closed $t$-support of $(X, f)$ is a left (resp., right) J-ideal of $X$ for all $t \in[-1, \alpha)$.

Proof. Let $x, y \in X$. If $y \in C_{\alpha}(f ; t)$, then $f(y) \leq t-\alpha$ and so

$$
f(x y) \leq f(y) \leq t-\alpha .
$$

Thus $x y \in C_{\alpha}(f ; t)$. Suppose that $x * y \in C_{\alpha}(f ; t)$ and $y \in C_{\alpha}(f ; t)$. Then

$$
f(\theta) \leq f(x) \leq \bigvee\{f(x * y), f(y)\} \leq t-\alpha
$$

by (3.1). Thus $\theta \in C_{\alpha}(f ; t)$ and $x \in C_{\alpha}(f ; t)$. Consequently, $C_{\alpha}(f ; t)$ is a left J-ideal of $X$ for all $t \in[-1, \alpha)$. Similarly we can prove it for the right case.

Theorem 3.14. For any $\mathcal{N}$-structure $(X, f)$ and $\alpha \in[\perp, 0]$, the following assertions are equivalent.

(1) The $\alpha$-translation of closed t-support of $(X, f)$ is a left (resp., right) J-ideal of $X$ for all $t \in[-1, \alpha)$.

(2) The $\alpha$-translation of $(X, f)$ is a left (resp., right) $\mathcal{N}_{\mathcal{J}}$-ideal of $X$.

Proof. Suppose that $\left(X, f_{\alpha}\right)$ is a left $\mathcal{N}_{\mathcal{J}}$-ideal of $X$ for $\alpha \in[\perp, 0]$ and let $t \in[-1, \alpha)$. For any $x, y \in X$, if $x * y \in C_{\alpha}(f ; t)$ and $y \in C_{\alpha}(f ; t)$, then

$$
\begin{aligned}
f(x)+\alpha & =f_{\alpha}(x) \leq \bigvee\left\{f_{\alpha}(x * y), f_{\alpha}(y)\right\} \\
& =\bigvee\{f(x * y)+\alpha, f(y)+\alpha\} \\
& =\bigvee\{f(x * y), f(y)\}+\alpha \\
& \leq t-\alpha+\alpha=t
\end{aligned}
$$

and so $f(x) \leq t-\alpha$. Thus $x \in C_{\alpha}(f ; t)$. Since

$$
f(\theta)+\alpha=f_{\alpha}(\theta) \leq f_{\alpha}(x)=f(x)+\alpha \leq t-\alpha+\alpha=t,
$$

for any $x \in C_{\alpha}(f ; t)$, we have $f(\theta) \leq t-\alpha$, i.e., $\theta \in C_{\alpha}(f ; t)$. Now if $y \in C_{\alpha}(f ; t)$, then $f(y) \leq t-\alpha$ which implies that

$$
f(x y)+\alpha=f_{\alpha}(x y) \leq f_{\alpha}(y)=f(y)+\alpha \leq t,
$$

that is, $f(x y) \leq t-\alpha$ for all $x \in X$. Hence $x y \in C_{\alpha}(f ; t)$, and therefore $C_{\alpha}(f ; t)$ is a left J-ideal of $X$.

Conversely, assume that the $\alpha$-translation of closed $t$-support of $(X, f)$ is a left J-ideal of $X$ for all $t \in[-1, \alpha)$. Suppose that there exist $a, b \in X$ and $t_{0} \in[-1, \alpha)$ 
such that $f_{\alpha}(a b)>t_{0} \geq f_{\alpha}(b)$. Then $f(a b)+\alpha>t_{0}$ and $f(b)+\alpha \leq t_{0}$, which imply that $b \in C_{\alpha}\left(f ; t_{0}\right)$ and $a b \notin C_{\alpha}\left(f ; t_{0}\right)$. This is a contradiction, and thus $f_{\alpha}(x y) \leq f_{\alpha}(y)$ for all $x, y \in X$. If

$$
f_{\alpha}(a)>\bigvee\left\{f_{\alpha}(a * b), f_{\alpha}(b)\right\}
$$

for some $a, b \in X$, then there exists $t_{1} \in[-1, \alpha)$ such that

$$
f_{\alpha}(a)>t_{1} \geq \bigvee\left\{f_{\alpha}(a * b), f_{\alpha}(b)\right\}
$$

which implies that $f(a)>t_{1}-\alpha, f(a * b) \leq t_{1}-\alpha$ and $f(b) \leq t_{1}-\alpha$. Hence $a * b \in C_{\alpha}\left(f ; t_{1}\right)$ and $b \in C_{\alpha}\left(f ; t_{1}\right)$, but $a \notin C_{\alpha}\left(f ; t_{1}\right)$, which is a contradiction. Hence

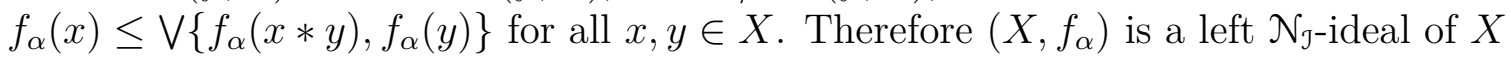
by Theorem 3.1 .

Given two $\mathcal{N}$-structures $(X, f)$ and $(X, g)$, we say that $(X, f)$ is a retrenchment of $(X, g)$ if $f \subseteq g$, that is, $f(x) \leq g(x)$ for all $x \in X$.

Definition 3.3. Given two $\mathcal{N}$-structures $(X, f)$ and $(X, g)$, we say that $(X, f)$ is a retrenched left (resp., right) $\mathcal{N}_{\mathcal{J}}$-ideal of $(X, g)$, denoted by

$$
\left.(X, f) \tilde{\subseteq}_{l}(X, g) \text { (resp., }(X, f) \tilde{\subseteq}_{r}(X, g)\right),
$$

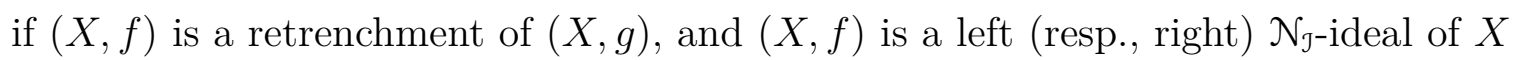
whenever $(X, g)$ is a left (resp., right) $\mathcal{N}_{\mathcal{J}}$-ideal of $X$.

Theorem 3.15. Let $(X, g)$ be a left (resp., right) $\mathcal{N}_{\mathcal{J}}$-ideal of $X$. For every $\alpha \in[\perp, 0]$, the $\alpha$-translation $\left(X, g_{\alpha}\right)$ of $(X, g)$ is a retrenched left (resp., right) $\mathcal{N}_{\mathcal{J}}$-ideal of $X$.

Proof. For any $x \in X$, we have $g_{\alpha}(x)=g(x)+\alpha \leq g(x)$. Thus $\left(X, g_{\alpha}\right)$ is a retrenchment of $(X, g)$. If $(X, g)$ is a left $\mathcal{N}_{\mathcal{J}}$-ideal of $X$, then Theorem 3.11 shows that $\left(X, g_{\alpha}\right)$

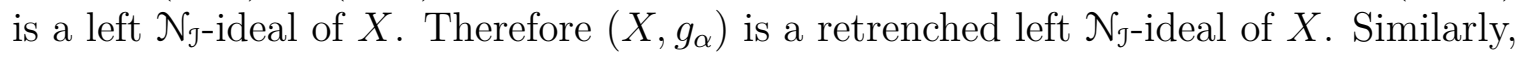
we can prove it for the right case.

Theorem 3.16. Let $(X, g)$ be a left (resp., right) $\mathcal{N}_{\mathcal{J}}$-ideal of $X$. If $\left(X, f_{1}\right)$ and $\left(X, f_{2}\right)$ are retrenched left (resp., right) $\mathcal{N}_{\mathcal{J}}$-ideals of $(X, g)$, then so is $\left(X, f_{1} \cup f_{2}\right)$, where $\left(f_{1} \cup f_{2}\right)(x)=\bigvee\left\{f_{1}(x), f_{2}(x)\right\}$ for all $x \in X$.

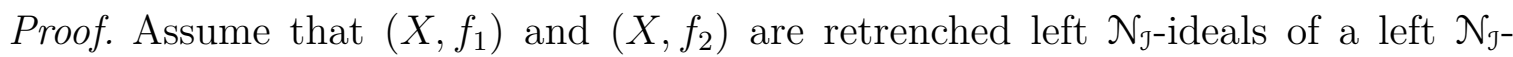
ideal $(X, g)$ of $X$. Then $f_{1}(x) \leq g(x)$ and $f_{2}(x) \leq g(x)$, for all $x \in X$. Thus $\left(f_{1} \cup f_{2}\right)(x)=\bigvee\left\{f_{1}(x), f_{2}(x)\right\} \leq g(x)$ for all $x \in X$, and so $\left(X, f_{1} \cup f_{2}\right)$ is a retrenchment of $(X, g)$. For any $x, y \in X$, we have

$$
\begin{aligned}
\left(f_{1} \cup f_{2}\right)(x y) & =\bigvee\left\{f_{1}(x y), f_{2}(x y)\right\} \\
& \leq \bigvee\left\{f_{1}(y), f_{2}(y)\right\} \\
& =\left(f_{1} \cup f_{2}\right)(y)
\end{aligned}
$$


and

$$
\begin{aligned}
\left(f_{1} \cup f_{2}\right)(x) & =\bigvee\left\{f_{1}(x), f_{2}(x)\right\} \\
& \leq \bigvee\left\{\bigvee\left\{f_{1}(x * y), f_{1}(y)\right\}, \bigvee\left\{f_{2}(x * y), f_{2}(y)\right\}\right\} \\
& =\bigvee\left\{\bigvee\left\{f_{1}(x * y), f_{2}(x * y)\right\}, \bigvee\left\{f_{1}(y), f_{2}(y)\right\}\right\} \\
& =\bigvee\left\{\left(f_{1} \cup f_{2}\right)(x * y),\left(f_{1} \cup f_{2}\right)(y)\right\} .
\end{aligned}
$$

It follows from Theorem 3.1 that $\left(X, f_{1} \cup f_{2}\right)$ is a left $\mathcal{N}_{\mathcal{J}}$-ideal of $X$. Therefore $\left(X, f_{1} \cup f_{2}\right)$ is a retrenched left $\mathcal{N}_{\mathcal{J}}$-ideal of $(X, g)$. The proof is similar for the right case.

Theorem 3.17. Let $(X, g)$ be a left $\mathcal{N}_{\mathcal{J}}$-ideal of $X$ and let $\alpha, \beta \in[\perp, 0]$. If $\alpha \leq \beta$, then the $\alpha$-translation $\left(X, g_{\alpha}\right)$ of $(X, g)$ is a retrenched left $\mathcal{N}_{\mathcal{J}}$-ideal of the $\beta$-translation $\left(X, g_{\beta}\right)$ of $(X, g)$.

Proof. Note that the $\alpha$-translation $\left(X, g_{\alpha}\right)$ and the $\beta$-translation $\left(X, g_{\beta}\right)$ of $(X, g)$ are left $\mathcal{N}_{\mathcal{J}}$-ideal of $X$ by Theorem 3.11. If $\alpha \leq \beta$, then

$$
g_{\alpha}(x)=g(x)+\alpha \leq g(x)+\beta=g_{\beta}(x),
$$

for all $x \in X$. Hence $\left(X, g_{\alpha}\right)$ is a retrenchment of $\left(X, g_{\beta}\right)$. Therefore $\left(X, g_{\alpha}\right)$ is a retrenched left $\mathcal{N}_{\mathcal{J}}$-ideal of $\left(X, g_{\beta}\right)$.

Similarly we have the following theorem for the right case.

Theorem 3.18. Let $(X, g)$ be a right $\mathcal{N}_{\mathcal{J}}$-ideal of $X$ and let $\alpha, \beta \in[\perp, 0]$. If $\alpha \leq$ $\beta$, then the $\alpha$-translation $\left(X, g_{\alpha}\right)$ of $(X, g)$ is a retrenched right $\mathcal{N}_{\mathcal{J}}$-ideal of the $\beta$ translation $\left(X, g_{\beta}\right)$ of $(X, g)$.

Theorem 3.19. Let $(X, g)$ be a left (resp., right) $\mathcal{N}_{\mathcal{J}}$-ideal of $X$ and let $\beta \in[\perp, 0]$. For every retrenched left (resp., right) $\mathcal{N}_{\mathcal{J}}$-ideal $(X, f)$ of the $\beta$-translation $\left(X, g_{\beta}\right)$ of $(X, g)$, there exists $\alpha \in[\perp, 0]$ such that $\alpha \leq \beta$ and $(X, f)$ is a retrenched left (resp., right) $\mathcal{N}_{\mathcal{J}}$-ideal of the $\alpha$-translation $\left(X, g_{\alpha}\right)$ of $(X, g)$.

Proof. It is straightforward.

A mapping $\varphi: X \rightarrow Y$ is called a homomorphism of IS-algebras if $\varphi(x * y)=$ $\varphi(x) * \varphi(y)$ and $\varphi(x y)=\varphi(x) \varphi(y)$, for all $x, y \in X$.

Let $\varphi: X \rightarrow Y$ be an onto mapping. Given an $\mathcal{N}$-structure $(Y, g)$, the $\mathcal{N}$-structure $(X, f)$, where $f=g \circ \varphi$, is called the preimage of $(Y, g)$ under $\varphi$. Given an $\mathcal{N}$-structure $(X, f)$, the image of $(X, f)$ under $\varphi$ is defined to be the $\mathcal{N}$-structure $(Y, g)$ on which $g$ is denoted by $\varphi(f)$ and is given by

$$
g(y)=\bigwedge_{x \in \varphi^{-1}(y)} f(x)
$$

for all $y \in Y$. 
Theorem 3.20. Every preimage of a left (resp., right) $\mathcal{N}_{\mathcal{J}}$-ideal under onto homomorphism is a left (resp., right) $\mathcal{N}_{\mathcal{J}}$-ideal.

Proof. Let $\varphi: X \rightarrow Y$ be an onto homomorphism of IS-algebras and let an $\mathcal{N}$ structure $(X, f)$ is the preimage of a left $\mathcal{N}_{\mathcal{J}}$-ideal $(Y, g)$ of $Y$. For any $x, y \in X$, we have

$$
\begin{aligned}
f(x y) & =(g \circ \varphi)(x y)=g(\varphi(x y)) \\
& =g(\varphi(x) \varphi(y)) \leq g(\varphi(y)) \\
& =(g \circ \varphi)(y)=f(y)
\end{aligned}
$$

and

$$
\begin{aligned}
f(x) & =(g \circ \varphi)(x)=g(\varphi(x)) \\
& \leq \bigvee\left\{g\left(\varphi(x) * y^{\prime}\right), g\left(y^{\prime}\right)\right\} \text { for all } y^{\prime} \in Y \\
& =\bigvee\{g(\varphi(x) * \varphi(y)), g(\varphi(y))\} \\
& =\bigvee\{g(\varphi(x * y)), g(\varphi(y))\} \\
& =\bigvee\{(g \circ \varphi)(x * y),(g \circ \varphi)(y)\} \\
& =\bigvee\{f(x * y), f(y)\} .
\end{aligned}
$$

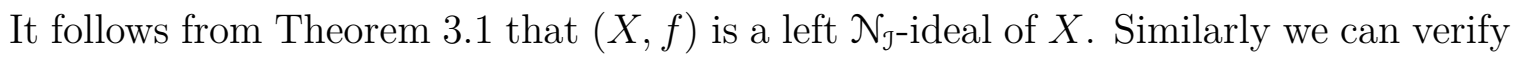
it for the right case.

Lemma 3.1. Let $\varphi: X \rightarrow Y$ be an onto mapping. Given an $\mathcal{N}$-structure $(X, f)$ and $t \in[-1,0)$, we have

$$
C(\varphi(f) ; t)=\bigcap_{t<s<0} \varphi(C(f ; t-s)) .
$$

Proof. For any $y=f(x) \in Y$, if $y \in C(\varphi(f) ; t)$, then

$$
\bigwedge_{z \in \varphi^{-1}(\varphi(x))} f(z)=\varphi(f)(\varphi(x))=\varphi(f)(y) \leq t .
$$

Hence, for every $s \in(t, 0)$, there exists $x_{0} \in \varphi^{-1}(y)$ such that $f\left(x_{0}\right) \leq t-s$. Thus $y=\varphi\left(x_{0}\right) \in \varphi(C(f ; t-s))$, and so $y \in \bigcap_{t<s<0} \varphi(C(f ; t-s))$.

Conversely, let $y \in \bigcap_{t<s<0} \varphi(C(f ; t-s))$. Then $y \in \varphi(C(f ; t-s))$ for every $s \in(t, 0)$, and hence there exists $x_{0} \in C(f ; t-s)$ such that $y=\varphi\left(x_{0}\right)$. It follows that $f\left(x_{0}\right) \leq t-s$ and $x_{0} \in \varphi^{-1}(y)$. Therefore

$$
\varphi(f)(y)=\bigwedge_{x \in \varphi^{-1}(y)} f(x) \leq \bigwedge_{t<s<0}\{t-s\}=t
$$

and thus $y \in C(\varphi(f) ; t)$.

Theorem 3.21. Every image of a left (resp., right) $\mathcal{N}_{\mathcal{J}}$-ideal under onto homomorphism is a left (resp., right) $\mathcal{N}_{\mathcal{J}}$-ideal. 
Proof. Let $\varphi: X \rightarrow Y$ be an onto homomorphism of IS-algebras and let an $\mathcal{N}$ structure $(Y, g)$ is the image of a left $\mathcal{N}_{\mathcal{J}}$-ideal $(X, f)$ of $X$. Let $t \in[-1,0)$ be such that $C(\varphi(f) ; t) \neq \emptyset$. Then

$$
C(\varphi(f) ; t)=\bigcap_{t<s<0} \varphi(C(f ; t-s)),
$$

by Lemma 3.1, and so $\varphi(C(f ; t-s))$ is nonempty for all $s \in(t, 0)$. Since $(X, f)$ is a left $\mathcal{N}_{\mathcal{J}}$-ideal of $X, C(f ; t-s)$ is a left J-ideal of $X$ and so the onto homomorphic image $\varphi(C(f ; t-s))$ of $C(f ; t-s)$ under $\varphi$ is a left J-ideal of $Y$. Hence $C(\varphi(f) ; t)$ is a left $\mathcal{J}$-ideal of $Y$. It follows from Theorem 3.5 that $(Y, g)$ is a left $\mathcal{N}_{\mathcal{g}}$-ideal of $Y$.

Acknowledgements. The research is supported by the Iranian National Science Foundation (Grant No. 96008529)

\section{REFERENCES}

[1] S. Abdullah and A. F. Ali, Applications of N-structures in implicative filters of BE-algebras, Journal of Intelligent \& Fuzzy Systems 29(2) (2015), 517-524.

[2] Y. S. Huang, BCI-algebra, Science Press, Beijing, 2006.

[3] Y. B. Jun, K. J. Lee and S. Z. Song, N-ideals of BCK/BCI-algebras, J. Chungcheong Math. Soc. 22 (2009), 417-437.

[4] Y. B. Jun, X. L. Xin and E. H. Roh, A class of algebras related to BCI-algebras and semigroups, Soochow Journal of Mathematics 24(4) (1998), 309-321.

[5] K. J. Lee and Y. B. Jun, The essence of subtraction algebras based on $\mathcal{N}$-structures, Commun. Korean Math. Soc. 27(1) (2012), 15-22.

[6] J. Meng and Y. B. Jun, BCK-algebras, Kyungmoon Sa Co. Seoul, 1994.

${ }^{1}$ Department of Mathematics,

Shahid Beheshti University, Tehran, Iran

Email address: Bordbar.amirh@gmail.com

${ }^{2}$ Department of Mathematics,

Graduate University of Advanced Technology, Mahan-Kerman, Iran

Email address: zahedi_mm@kgut.ac.ir

${ }^{3}$ Department of Mathematics Education,

Gyeongsang National University, Jinju 52828, Korea

Email address: skywine@gmail.com

${ }^{*}$ Corresponding Author 